\title{
1 Psychology and Individuals
}

My Preface declared my target for study: collectivities and social behavior. What can psychology, given its ordinary focus on the individual, tell us about population groups of one kind or another? My aim in this first chapter is to identify points of similarity between the individual and the collectivity, with the hope of transferring psychology's insights about the former to the latter. This we commonly do in discussing national character or stereotypes of a class, ethnicity, occupation, or the like.

The difficulties are obvious. To begin with, the psychologist can look inside individuals in ways not available to historians, simply because historians' subjects are all beyond the reach of questionnaires, focus groups, and assorted laboratory tricks. Yet if a record of group action remains, of course inferences can be drawn about tendencies and character. Psychology may suggest rules of prediction to guide such inferences, if something discoverable about individuals can be shown always or generally to have certain results - in short, if there are behavioral consistencies about which the discipline is agreed. In this way not only single individuals can be better understood but a number of individuals together, constituting a body of a size sufficient to have made a difference.

As to consistencies, these must depend on the raising of the psychological discipline to the level of a science, able to discern and define the invariant aspects of personality and fit them together in general statements. Hopes of attaining this goal have animated discussion from well back in the history of the social sciences but, to consider only recent times, we have the recollection of Hazel Markus and Shinobu Kitayama, that "the social psychology of the 1980s was very inclined to universals. Universalism was a sign of commitment to science, and the goal of science was to pursue the universal laws of human nature". ${ }^{1}$

Within psychology, no specialty would appear to be more relevant to my purposes than the study of values, where Robert Hinde is well known for his research. "Some norms, beliefs, and values," he tells us,

\footnotetext{
may be shared by most or all members of a society. It is to the latter that the term 'culture' is usually applied. Briefly, 'culture' refers here to the ways in which human groups or subgroups differ that are communicated between individuals, with special reference to beliefs, values, and behaviour. 'Culture' is thus best viewed as existing in the minds (separately or collectively) of the individuals in a group. There is thus a continuum between the culture of a given society and the beliefs and values of the component individuals. It is unfortunate that the former has become the province of sociologists and anthropologists, the latter of social and developmental psychologists
}

1 Quoted, Markus and Kitayama (2003) 280. 
- and he continues with remarks about cross-cultural studies of upbringing and acculturation which bring out the differences from one society to another. ${ }^{2}$ The reminder here, that the boundaries between individual and collectivity are almost meaningless, serves me in reviewing the relevant lines of research in recent generations.

The results have not been so helpful as might be expected. How the beliefs and traits of communities play out across time and thereby shape the story of a population is of course endlessly analyzed in the service of educational, penal, economic, public health, or other categories of public policy. The object of study may be a caste, a profession, a class or income level (all, of course, contemporary). In contrast, long-term predictions about individuals, so common in high-school yearbooks and a thing we all take for granted, have run into research problems that invite an explanation.

Where, as it seemed, the approach had been loose and intuitive a century ago, something more scientific was surely possible. One improvement might be found in convincingly large groups of subjects and the translation of test-findings into numbers. Working with groups diminished the risk of conclusions based on exceptional or unrepresentative subjects, and numerical measurement allowed for closer argument and comparison.

A well-known study was Lewis Terman's sampling of some 1500 brighter-thanaverage children from 1922 on to his death in 1956 and, by continuation under others in research, for decades thereafter. The result could be regarded as a great cluster of tiny histories, however narrowly focused. Terman began with only human intelligence as his target; but in the search for the origin and nature of that advantage, he cast a very wide net. Parents and teachers were invited to describe children under headings like carefulness, anxiousness, sociability, and dozens of other qualities. These could in time be correlated with life-outcomes. Better perhaps than brainy, the chosen children proved also happier and more successful than the average, in the long run. ${ }^{3}$

Alternatively, the focus could be further narrowed to yield more exactitude. "Aggressiveness" in little children could be described by peers, teachers, or parents and measured in various tests. It might show up in many sorts of antisocial behavior, even criminal, twenty years later; and this could be verified. Or "neuroticism" could

2 Hinde (1996) 369; (2002) 20, "the basic psychological characteristics to which I refer are at the individual or near-individual level"; and repeated, "culture is best viewed as existing in the minds of individuals". Compare Hofstede (2002) in an interview, "you have to realize that culture is a construct. When I have intelligent students in my class, I tell them, 'One thing we have to agree on: culture does not exist.' Culture is a concept that we made up which helps us understand a complex world, but it is not something tangible like a table or a human being. What it is depends on the way in which we define it”.

3 On the need to consider both cognitive and non-cognitive factors in individuals' life development, including both their material success and their personality traits, see, e.g., Jackson (2006), Hall and Farkas (2011), or Friedman and Martin (2011) 7ff., 68. 
be rated in a sample of young couples before their marriage and, decades later, it corresponded with the likelihood of success in their marriage. ${ }^{4}$ The effects of conscientiousness, measuring how you perform a task even when no one is checking up on you, could be traced in a representative sample of many thousands of American children across many decades, to see what its consequences were (and they were good, of course); similarly, responsiveness to authority in the late teens and early twenties was found to correspond significantly with much later feelings of career satisfaction and contentment in life; and there are many other examples of traits that affect the whole course of life. $^{5}$

The studies just instanced belong to the 1980s and later. They represent more or less current practice. Before that date, however, over a period beginning in the 1960s, such research confronted serious criticism. The problem lay in the prevailing ambition to make psychology not only scientific but rigorously so - like physics, the ideal. The discipline should aim at unified statements holding true over masses of data, the bigger the better; it should aim at laws of Newtonian purview, at theories of Einsteinian compression and parsimony. ${ }^{6}$ It should employ only sharply defined terms and exact measurements to allow verification and replication.

By 1964, however, criticism of even the most generally accepted trait- and personality-studies dismissed them all as "a dead end". They had no statistical validity. ${ }^{7}$ A further assault of book length was delivered by Walter Mischel a little later (1968). This work was a very careful and forbiddingly technical evaluation of personality research to date. At the end, the author thought it "not surprising that large-scale applied efforts to predict behavior from personality inferences have been strikingly and consistently unsuccessful." Predictions about what a person would do, if asked, in a variety of imagined situations that would reveal character traits, he judged entirely unreliable. Correlations across them all fell below a level of any significance, leading him "to clear conclusions. With the possible exception of intelligence, highly generalized behavioral consistencies have not been demonstrated, and the concept

4 Magnusson (1988) 92, 109, 119-30, tracking early aggressiveness and later criminal problems, and 156, consensus on the correlation between "early aggressiveness and adult criminality"; Bergman and Magnusson (1990) 5f.; and Brody (1994) 420f.

5 On conscientiousness, see Segal (2012) 1438f., 1442, 1453; and other studies, e.g., Winter et al. (1981) 48, 95 or Vaillant (1983) passim, on "maturity of ego defenses" measured across decades.

6 E.g., Tooby et al. (1992) 30 on parsimony ("The goal, as in physics, is for as few principles as possible to account for as much as possible”); also, aim at reduction of variables through taxonomies, Malle and Dickert (2007) 1012; and the declared purpose is "to build a science of the person," "a metadiscipline”, working off “meta-theories”, as proposed by Mischel (2004) 1, 13, 18.

7 "Dead end," the verdict of Philip Vernon, a student of Gordon Allport, recalled by Mischel (1968) 146, 296; and the "dead end" sensed in sociology in the 1980 s as a consequence of logical positivism, cf. Mommsen (1989) 121. 
of personality traits as broad response dispositions is thus untenable." ${ }^{8}$ The verdict amounted to the dismissal of "personology" and its research literature, wholesale.

Personality-study had been given essential form and standing by Gordon Allport in the generation prior to Mischel. Now to take it on in this all-out fashion as Mischel did was bound to attract attention. At first his work was received with alarm; its impact was said to have produced "a crisis" in the research community. ${ }^{9}$ As its argument sank in, Seymour Epstein saw the consequences: "The charge that personality traits do not exist clearly strikes at the very heart of personality theory. One could well argue that if individuals do not have relatively stable behavioral dispositions that differentiate them from other individuals, then the concept of individuality itself can be dispensed with."10

Epstein in two articles written as a pair under the title, "The stability of behavior: on predicting most of the people much of the time" (1979-80), went on to make clear one point. This he pressed home with overwhelming statistical support: an individual's traits, detected in response to a variety of situations, scenes, and descriptive phrases, might vary from one to another but not across the board. If the data were aggregated, as aggregation rose, so did over-all consistency.

In challenging Mischel's verdict, what Epstein argued on a theoretical or even philosophical level could only seem self-evident to a layman. ${ }^{11}$ We simply cannot imagine that people we think we know will do something they've never done before, tomorrow, and will try something else equally strange to their routines, the day after. What we have observed them to do is what they are; their tendencies direct and define them; they are no more likely to change in some radical way than their body to grow a third arm; and while personality may allow an occasional "departure", something "quirky", nevertheless our choice of such descriptive terms testifies to our belief in a stable identity at each person's core.

In one of his articles Epstein made reference to a huge trait-study (on honesty, undertaken in the 1920s) that looked at many thousands of children across many years. The researchers had explained that a lack of correlation indeed showed up in tests, just such as Mischel was to point to much later; but the reliability of these

8 Mischel (1968) 146.

9 On Allport's role, see, e.g., Caspi (1998) 312 or Hogan (2009) 249. Mischel's book "strikes at the very heart of personality theory," so, Epstein (1979) 1098; but, while its impact is "an incredible achievement", it has constituted "a major impediment" to progress in the field, so, Roberts (2009) 137f. "The most daring challenge," in the words of Caspi (1998) 313, it "dealt a heavy blow to the trait approach," Schütz and Vater (2007) 995; thanks to Mischel and another researcher, "trait psychology continues to be marginalized," Costa and McCrae (2009) 299; and the field is pronounced in "crisis", Epstein (1980) 790 and still in Costa and McCrae (2008) 179.

10 Epstein (1979) 1098.

11 As Allport insisted, quoted in Caspi (1998) 312f. On animal individuality, a lively field, see, e.g., Bergmüller and Taborsky (2010) or Cote et al. (2012) 1472ff. on the western mosquitofish. 
tests would be increased to a probative level if they were multiplied. "Just as one test is an insufficient and unreliable measure in the case of intelligence, so one test of deception is quite incapable of measuring a subject's tendency to deceive. That is, we cannot predict from what a pupil does on one test what he will do on another." ${ }^{\text {"I }}$ It was in line with this dictum by a predecessor that Epstein and others thus recommended a "cross-situational" approach, as it could be termed. It offered distinct advantages over the deceptively precise laboratory measurement of single moments or stimuli. ${ }^{13}$

Epstein's views seem to me doubly interesting. Not only had Mischel been quite mistaken but beyond that, prediction about most but not all people, and about most but not all of the time, was something to be taken seriously even though it might be proposed only in words not numbers. That possibility would have been approved by William Cameron, reminding students of sociology long ago, "Not everything that can be counted counts, and not everything counts that can be counted." ${ }^{14}$ Human behavior may be just too complicated for numerical treatment, of which the aims are so easily lost among variables and terms of measurement irremediably subjective. "Science", that ennobling term, may hold out ideals of exactitude that simply do not fit the problems.

On the other hand, there was no need to abandon two essentials. These were the existence of the individual personality and its most usual but not complete or invariable stability. Longitudinal studies that took these essentials for granted, implicitly if not explicitly, could go right on; and in fact, so they did. ${ }^{15}$ Their total in fact now reaches into the hundreds.

Mischel himself contributed in a most unpredictable way. He, like his great predecessors in the discipline, William James and Sigmund Freud, had developed an interest in our powers of self-control. Self-control involves two or more psychological attributes in distinct, even antagonistic roles, inside our heads. This invited investigation. Mischel had published a dozen more or less relevant studies when, in 1968,

12 Epstein (1979) 1101 on the 1920s study, and the general reminder (1102) that "a trait is a generalized tendency" which "does not imply... trait-relevant behavior in all situations or even on all occasions in the same situation"; further (1980), amplifying his arguments and pointing out the mismatch between the possibility of exact replication in the hard sciences that sets a standard unattainable in the social sciences.

13 Epstein (1984) 210 recalls the reception of his views; further, Magnusson (1988) 4.

14 Quoted, Cameron (1963) 13 (he had proposed a part of the same witty doctrine years earlier; the two-part whole has often been misattributed to other persons).

15 Young et al. (1991) list 213 studies, at a rising rate of increase (pre-1950, only 19). For the continued or revived belief in the (not perfect) stability of personality, see the positions taken, e.g., by Magnusson (1988) 55, 65, Mischel et al. (1989) 933, Aken and Asendorpf (1996) 205-08, Caspi (1998) 312, or Friedman and Martin (2011) 6. Berry et al. (2002) 98 defend Mischel's 1968 dismissal of stable personality while ignoring all of Epstein's work; disregarding the probative value of the longitudinal studies of self-control; and supposing that assessments of stability in traits by one's self are the only sort possible, and may be discounted, without mention of assessments by knowledgeable other parties. 
he subjected to irresistible temptation a large sample of four-year-olds in the nursery attached to Stanford University. Singly, the children were offered a reward, a marshmallow, but told they could have two if they could wait a little bit and then ring a bell to call back the research-person. Being left alone in the test-room but observed behind a one-way window, they could be watched and the minutes, even the seconds, could be counted before they made their choice. They did delay and the effort of selfcontrol could be exactly calibrated; but after a few minutes most of them surrendered to the irresistible presence of the single reward. ${ }^{16}$ It was a neat, intriguing experiment. It could be described by its author (2011) as "now classic", and similar longitudinal self-control studies continue to date. ${ }^{17}$

Forty years post-facto Mischel recalls how idle conversation with his own children over the kitchen table had once made him wonder, What ever became of those testchildren as they grew up? This excellent question occurred in the wake of Epstein's articles, which had perhaps not gone unnoticed. So, beginning in 1981, Mischel got in touch with his test-subjects of 1968 to conduct follow-up outcome-studies. Did children's ability to resist instant gratification indicate, as surely it must, a trait of character, willpower, which as they grew up would register in desirable successes? Did it make for a planful person, a prudent one, with good results? ${ }^{18}$ Such questions, taking for granted a notion of personality very different from that proposed in Mischel's 1968 book, led rather to the traditional view of a "basic structure of personality," which, as he said, "underlies the individual's uniqueness" and persists across time. ${ }^{19}$ It could be profitably examined through "situationist" questions, just as had been pointed out in the 1920s. The author, in a very abundant flow of publication citing and refining his own past work, gradually edged toward acceptance of the new, or not so new, situational orthodoxy. In the end he seemed to claim it for his own and even earned a tribute of relevant papers in a research journal's honorific volume (2009). ${ }^{20}$

16 Bandura and Mischel (1965) 698 and passim for previous articles by Mischel and many also by the senior researcher, Bandura; acknowledgement of studies by William James and Freud, 704, and Mischel et al. (1989) 934. The test itself has been often described, e.g., by Metcalf and Mischel (1999) 3f., Lehrer (2009) 26, or Mischel et al. (2011) 252, and is a standard one (not with marshmallows) among biologists using rats, cf. chap. 3 at n. 84 .

17 Closely similar measurement of delay before cheating, among other things, in Mischel and Gilligan (1964) 412ff. or Nunner-Winkler (1999) 262; “classic,” Mischel et al. (2011) 252; Moffit et al. (2011) 2695ff., >1000 New Zealand children tested for self-control and its consequences over three decades. 18 On Mischel's recollections, and testing for planning habits and care, see Mischel et al. (1988) 687 and Lehrer (2009) 27.

19 Quoted, Mischel and Shoda (1995) 254; 246, on "the invariance of personality" in the face of "variability across situations"; Mischel et al. (1988) 693, on "enduring personal qualities", and Mischel (2004) 3, on "stable overall individual differences".

20 “Cross-situational”, e.g., Brody (1994) 420; Hogan (2011) 249, recalling Allport's acknowledgement of the flexibility of the personality, as opposed to the "situationists criticizing a non-existent claim" that personality was utterly inflexible; Mischel (1968) 295f., rejecting Allport's misleading doctrines in 
Trait-defining before measuring trait-effect might look like this, for "conscientiousness": ${ }^{21}$

\begin{tabular}{lll}
\hline Adjectives & Adult Q-Sort Items & Child Q-Sort Items \\
\hline Organized & Dependable, responsible & Persistent in activities, does not give up easily \\
\hline Planful & Able to delay gratification & Attentive and able to concentrate \\
\hline Reliable & Not self-indulgent & Planful; thinks ahead \\
\hline Responsible & Behaves ethically & $\begin{array}{l}\text { Reflective; thinks and deliberates before spea- } \\
\text { king or acting }\end{array}$ \\
\hline
\end{tabular}

Subsequent evaluation of subjects under such headings could claim a degree of uniformity if the definitions were well fixed. Descriptive phrases could support single words, most often adjectives; these latter could be clustered together in turn to make up the meaning of a trait-word. Specifically and under controlled lab conditions, the child who did well on the marshmallow test could plainly think ahead and thus possessed, or demonstrated, or fitted under, "conscientiousness". Illustrated in such ways, a science of personality allowing reliable prediction of outcomes would have great practical use.

Could the science tell us about Homo sapiens everywhere and across all time? We would certainly like to know. It was a point of weakness in the claim, however, that terminology can show only what people say they understand when they hear such a word as "conscientiousness", defined by descriptive phrases generated in a given, living speech community. The truth is as old as Protagoras, declaring "Man is the measure of all things" but going on to explain that "things are to you such as they appear to you, and to me such as they appear to me."22 To bring such relativism up to date, we have only to ask whether a "conscientious" person would turn up for appoint-

this regard; acceptance of the need to mix situation and tendency, often by Mischel, or Shiner (2011) 270 or Hill and Lapsley (2009) 245; so also, for acknowledgment of "aggregation" and credit but also some misperception of Epstein, and the citing of Mischel's own position of 1982, see Mischel (1984) 285, Mischel and Shoda (1995) 246, Mischel (2004) 3f., and Mischel (2007) 267; Mendoza-Denton and Mischel (2007) 176, now explaining Mischel's 1968 work on the "personality paradox" in terms of situations, dismissing Epstein and aggregation; Epstein and his citing of the 1928 Newcomb longitudinal survey, credited in Mischel (2004) 2f.; and Mischel's retreat noted, Schütz and Vater (2007) 995. Beyond the celebratory volume, there were other Festschriften, cf. Donnellan (2009) 117.

21 Caspi (1998) 317, part of Table 6.1, "Examples of Trait Adjectives... defining the Big Five Factors"; for "conscientiousness" among the Big Five see Pervin (1994) 103f., 108, or Löckenhoff and Costa (2007) 115; in longitudinal studies, conscientiousness is a prime agent in longevity, see Friedman and Martin (2011) 9, and in economic success, see Segal (2012) 1439.

22 Plato, Theaetetus 152A, trans. Jowett. 
ments on time - and not ten minutes late? Certainly not a full half-hour? ${ }^{23}$ Or will that person pay bills on time? Does that mean within a week? A month? Two months? And will the conscientious person also care for a ne'er do well second cousin for a week, or over the course of an indefinite illness? Or can't one count on that? Answers will be specific to the respondents' particular culture, each with its own descriptive words and values, whether in New Haven or New York, or in Tangiers or Amman - to say nothing of bygone times and places in which historians are interested.

And as a further difficulty, "trait testers appear to assume that whatever they name has objective reality; many need not so much to improve the measures but to improve or change their thought regarding traits." 24 Indeed "conscientiousness" has never been seen or heard or touched - only moments of behavior that might among Anglophones be tagged with that particular phoneme. In reply to this objection on the logical plane, against what philosophers call "realist" interpretations, it could be pointed out that quite similar studies have been made of the lexicon of colors, recognizing that each of the terms represents a semantic pause not a physical one along the spectrum. The pauses differ in number and position in different languages but the resulting terminological ambiguities can be controlled through spectroscopy and wavelength-nanometers. These are quantifiable realities out there for all to see. But alas, no such science can be applied to the lexicon of traits. They remain both subjective and culture-specific, even where the research-objective is the description of human behavior in general.

If the logical problems have not really been addressed, though occasionally noted, at least ethnocentrism can be reduced or corrected.

Cross-cultural studies have been most conveniently directed at ethnic sub-groups within the United States (Asian, African, or other); but also in other countries, overwhelmingly in the West (Iceland, Sweden, the Netherlands, Germany). ${ }^{25}$ Even in this limited region results have shown differences not reducible to uniformity. Study could be extended to East Asia, for example, through a test to determine if shyness in a child

23 "Large differences" in ideas of punctuality comparing Estonians, Moroccans, and Americans, noted in White et al. (2011) 486; Brody (1994) 420, on punctuality as a subset of conscientiousness, in Mischel's treatment.

24 Lehman and Witty (1946) 490, or Harré (1986) 4, making the same point against realism, where psychologists misrepresent emotions through supposing "there is something there" that is the phenomenon, rather than "angry people, grieving families", etc. What we mistake for the object of study is only a word, which in the language of each culture designates something particular to that culture. "We reify and abstract from that concreteness at our peril." Further, below, n. 48.

25 U.S. sub-groups, Asian- or African-Americans in Caspi (1998) 318; Oishi et al. (2008) 307 and passim; Okazaki et al. (2009) 378; German children, Asendorpf and Aken (1999) 817, 831, "predictive of important developmental outcomes in both the cognitive and social domain"; Asendorpf (1999) 227; Bergman et al. (2003) 136f.; and New Zealanders, above, n. 17. In all of these studies the entirety of the population is not well sampled. 
turns into a general inability to handle relations with others, amounting to "social incompetence", by which marital and occupational success will be later jeopardized. On this score, in Japan, a style of upbringing would be approved which by American standards would likely handicap a child in adult years. The definition of the trait and the word for it turns out not to be universal. Or consider whether a child, seen by peers, parents, teachers or trained observers as prone to quarrel and bully, would likely get into trouble of some form during the teens and early adulthood. ${ }^{26}$ Yet there are worlds where anything else would count as a costly, undesirable weakness. Illustrations of this can be found in the next chapter.

How then could trait research lead to "a generalizable and comprehensive representation of personality trait structure”, true of all our species? ${ }^{27}$ An answer might be sought through a lexical approach - through English, that gigantic conglomerate, so widely spoken. From Gordon Allport's days in the 1930s, people competent to explore it began to pull out words that designate traits, at first identifying close to twenty thousand and reducing this total by amalgamating synonyms so far as these could be agreed on (though anyone acquainted with language history can see how approximate this business must necessarily be). Thus by repeated efforts in successive publications the twenty thousand could be reduced to less than five thousand and then to a mere thirty and at last, in recent decades, still further. The resulting "Big Five" seem now well established. They include neuroticism, extraversion, openness, agreeableness, and conscientiousness, as some say; or the first three of these and culture as a fourth, and several other possibilities for a fifth. Or in preference, the Five should really be Six or Three or Two; or Thirteen. ${ }^{28}$ Some limited number of these have been

26 Asendorpf and Weinert (1990) 194f.; Aken and Asendorpf (1996) 206ff., 214.

27 “Trait psychology has been a long quest for a universal taxonomy of traits," so, Mischel (1968) 43; quoted, Caspi (1998) 318 (with my italics), cf. 320, and passim.

28 On Allport's work, see, e.g. Saucier and Goldberg (2006) 268, in the best general account of traitlexical studies; on the origins of the Big Five, see Smith et al. (2006) 130f.; dependence of the Big Five on English, Zhou et al. (2009) 364; Mischel (1968) 45 (“Culture” included), 58 (traits "involve broad categories with vague semantic referants”); Pervin (1994) 103f.; Ozer and Benet-Martínez (2006) 404 (culture); Löckenhoff and Costa (2007) 115; Schütz and Vater (2007) 994; Caspi (1998) 316 (reduction in numbers of traits, to three); three, six, or seven traits, perhaps, say Winter et al. (1998) 233; lack of the fifth factor in various languages, or variants of all five, Smith et al. (2006) 131; reduction to two, Zhou et al. (2009) 367; and Saucier and Goldberg (2006) 274 and Zhou et al. (2009) 365, on the Six proposed. For Thirteen, to accommodate marked differences in Mexican and Israeli sampling-results, see Diaz-Guerrero and Diaz-Loving (1994) 134; 130, noting the "fascinating" (indeed!) acceptance of U.S. ethnocentrism; an overview minimizing any weaknesses in the Big Five model, in Berry et al. (2002) 92-98, while acknowledging that the Big Five model, formed solely in the USA, needs another superfactor (Asian) to fit test data; and this seems to me equally "fascinating". On the IBM model and Hofstede, Nohria et al. (2008), by questionnaire among many hundreds of very big business employees, finds [only] a 60\% fit in "overall motivation" with four "drives" recalling the Big Five; and these are "hardwired" and explain "everything we do" as a species. The study illustrates how much in fashion are such studies; and, oblivious of all the above, Terraciano et al. (2005) 96 find "most 
called "superfactors" (and with equal truth, also "psycholexical artifacts"), proposed as the essential elements in human personality. ${ }^{29}$ Granted, they do not explain anything, as has often been pointed out. They only describe. ${ }^{30}$

For their study and definition, the lexicon was English. Here lay the likelihood of error. "The danger of universalism," meaning what it is that all cultures share, "is that it can enshrine as universal understandings those that are in themselves culturally limited." ${ }^{31}$ Gerard Saucier, long a leader in personality factor analysis, saw the need to expand the search to include other languages. A number of these in recent years had been subjected to analysis (Polish, Croatian, and others), to which Saucier with colleagues added Turkish, Greek, and Chinese. ${ }^{32}$ The Chinese team found that a personality model in which Seven factors were imagined seemed to fit the evidence best - better than Five, and consisting of Conscientiousness/Diligence, Extraversion (these two posited in the Big Five model), Unselfishness, Negative Valence, Emotional Volatility, Intellect/Positive Valence, and Dependency/Fragility. The Seven are listed here to give an idea of the highly elastic quality of such hypothesized elements in personality, being no different in this regard from other proposed "Big" traits, all as inclusive as possible and, to that extent, imprecise. ${ }^{33}$

Another ambitious recent survey enlisted the cooperation of psychologists and their students in no less than fifty nations across six continents. The college students used as raters were asked to provide a profile of someone they knew well, under many descriptive headings, and their answers were analyzed to see how well these fitted with the Big Five model. A notable approximation to uniformity was asserted, although in the face of very substantial differences in gender issues and perceptions reflecting "a host of culture-level variables that differentiate Europe from Asia and Africa." At the end, claims that "the data largely confirm recent findings of universality in trait psychology" and "the biological basis of personality traits", do seem excessive. ${ }^{34}$

personality psychologists today agree that the dimensions of the five factor model (FFM)... account for the covariation of most personality traits".

29 On these quoted words, see Caspi (1998) 316.

30 For the Big Five model as descriptive not explanatory, see, e.g., Pervin (1994) 109 or Costa and McCrae (2009) 299; and the Five have "no theoretical base”, so, Saucier and Goldberg (2006) 278.

31 Kapferer (2002) 4.

32 Saucier and Goldberg (2005) 271 and Zhou et al. (2009) 364f., where a number of discrepancies between the non-English and Big Five results turned up; and 366 (Filipino, Hebrew) and passim (Chinese).

33 Zhou et al. (2009) 363; a preference for a Nine-factor model, not Five, in Diaz-Guerrero and DiazLoving (1994) 137.

34 McCrae and Terracciano (2005) 548, the non-Western raters generally "Westernized"; 550, raters choosing generally to profile their peer group, cf. Tables 1-2 showing two-fifths of the site-list in Europe and $89 \%$ of persons profiled of age $18-25,94 \%$ of age $18-30$; 554f., an enormous range of gender contrasts, from Nigeria to England; 553, deviations in Africa suggesting "some distinctive African 
A weakness in this particular survey as more generally, too, was the narrowness of the data-base. Psychologists, not only in the United States, have always been heavily dependent on the enrollments in their very own lecture courses, even though "college students are not, in general, representative of their national populations". The fact is acknowledged (though rarely).$^{35}$ College students in the United States have represented only a luckier or more determined quartile, and in numbers further tilted in this or that direction by economic class and ethnicity; by their very age-group they are only themselves, not an entire population. ${ }^{36}$ A degree of myopia that tolerates dependence on this test-sample and largely dispenses with history and anthropology seems to pervade the discipline even in discussions about our species generally. ${ }^{37}$

personality structure that differs appreciably from [that] found elsewhere in the world"; 559, quoted, "confirm... biological"; further, Optimism not Openness; and Terraciano et al. (2005) passim, condensing McRae and Terraciano without change. Ozer and Benet-Martinez (2006) 404 show that links between two of the Five (Neuroticism and Extraversion) are moderated by culture, while Lehman et al. (2004) 698 show optimism itself, and pessimism, differing in East Asian and American samples; and Okazaki et al. (2009) 382 on comparison of the American Big Five bipolar factors with those of a Chinese version, show a match under only one of the Five (which was not among the three most widely accepted members of that Five).

35 Perhaps needlessly to show the pervasiveness of college students as samples, I instance D’Andrade's study of 1965 in Mischell (1968) 46; Winter et al. (1981) 95; Winter et al. (1998) 239, referring to studies of the 1950s to 1970s; Kahneman and Tversky (1982) 167f.; Vaillant (1983) 344f.; Epstein (1984) 223; Triandis (1989) 512; Miller et al. (1990) 35; Mischel and Shoda (1995) 250; Triandis (1995) 122, 202, and passim; Guimelli (1999) 14; Mischel (2004) 12; McRae and Terracciano (2005) 548; Terraciano et al. (2005) 98; Rohan (2007) 1010f.; Liem et al. (2009) 223; White et al. (2011) 483f.; Kahneman (2011) 403; or Keith (2011) 24f., 27. Seeing the sampling as a weakness are Markus and Hamedani (2007) 9; Oishi et al. (2008) 309, 312, or Shweder and Sullivan (1993) 498 to the same effect, on "one particular population (e.g., the contemporary secularized Western urban white middle class)"; more conclusive, Haidt et al. (1993) 625 and passim. In the social sciences generally, for students as approximately 80 per cent of respondents, see below, chap. $2 \mathrm{n} .64$; to be noted is the caution of Stanovich and West (2000) 664, note 2, explaining their use (648) of a range of standard tests of cognitive capacity: "All the work cited here was conducted in Western cultures which match the context of the tests. Of course, we recognize the inapplicability of such measures as indicators of cognitive ability in cultures other than those in which the tests were conducted;" and more plainly, Higgins et al. (2008) 175, "the participants in our studies are college students. Thus, the bad news is that no claim can be made that our samples are representative of the general population of each nation" (Australia, China, Israel, Italy Japan, United States). For the quoted words in my text, "students not, in general, representative of their national populations," see McCrae and Terracciano (2005) 548; similarly Okazaki et al. (2009) 386 on personality assessments cross-culturally, warning of "a critical shortcoming... [samplings] primarily in university settings”, see also 378, or McSweeney (2002) 94, or Smith et al. (2006) 267, "university students... hardly representative".

36 Indication of the importance of age-group is clearer among non-Westernized populations than among U.S. students, see Mishra (1994) 225, 235 f.

37 Farr (1984) 126, "psychologists are ignorant as they only rarely read the literature of social sciences other than psychology". 
As an alternative, however, and to reach out more widely, a kind of participant quite different from American college students could be studied. In the 1970s and subsequently Geert Hofstede turned to the results of a very long questionnaire imposed not long prior on more than a hundred thousand of its employees by the International Business Machine company. The object of this device had been improvement in management policies all over the world: essentially to understand and increase job-satisfaction. It amounted to a gigantic data-base, any use of which for statistical evaluation of personality types was bound to be taken seriously. Hofstede thought he could discern in the data a small number, at first four and then five, of "dimensions" such as "collectivism-individualism" and "masculinity-femininity", in terms of which the whole of any person's behavior and inclinations could be summed up. Differences across countries could be perceived, yielding profiles of national character in quantifiable degree; and the same "dimensions" could be looked for and studied in all sorts of other, smaller samplings, by other researchers, following on his success. A favorite target of research has been the contrast at the center of the dimension "individualism-collectivism" and its expression in the way the typical American, and the typical Asian, act and see themselves. ${ }^{38}$

To illustrate the kind of project inspired by Hofstede, one of some scores of questions in a survey will serve, asking respondents:

You're starting a new business, and you are looking for a partner. Which is the most important factor in choosing a partner?

A. Someone with the same business interests

B. Someone who has been successful in previous business ventures

C. A close friend

D. A senior, successful, experienced member of the community

Answers were interpreted as showing, (A) so-called horizontal individualism, HI; (B) vertical individualism, VI; (C) horizontal collectivism, HC; and (D) vertical collectivism, VC; and these were in turn believed to show what Hofstede had explained as societies in which the individual floats free, so to speak, and looks around for help among his peers or others according to the needs of the moment, or instead, feels himself or herself from birth a member of a group to which loyalty and respect are owed, either at the peer level or in the hierarchy. ${ }^{39}$

Hofstede's books have been much admired and described by their author himself as producing "a paradigmatic shift in cross-cultural studies". His results nevertheless have come in for scattered criticisms which Brendan McSweeney capped off in 2002

38 See below, chap. 3 at nn. 135ff, and chap. 4 at nn. $58 \mathrm{ff}$.

39 For the question, see Triandis (1995) 210; for the meaning underlying the four answers, see a convenient condensation of Hofstede on individualism-collectivism in Kim et al. (1994) 2. 
with a full-scale appraisal..$^{40}$ In brief, the whole edifice of interpretations could be brought down by a careful look at its various parts, of which those that seem weakest to a historian are the quite inadequate nature of the sampling, and the "stories" that Hofstede proposed to illustrate his ideas. The choice of respondents was really no more representative of the larger surrounding communities than an equal number of college students would have been (and in many countries, too, the number of respondents was very small). The illustrative stories raised further problems: for example, showing how only the Austrian national character, and by itself alone (if it could be known in the relevant period), could have produced Freud and his theories - an idea no historian would agree to. ${ }^{41}$

Attempts continue to discern some governing pattern in human behavior. They extend from the way individuals are commonly observed to act (and so are judged to have some certain "trait"), to their motivating "values", "beliefs", or "principles". These last three terms are quite conventionally treated as synonymous by Shalom Schwartz in recent studies.

It was Schwartz' aim “to identify the structure of value relations”, those "guiding principles" by which individuals in dozens of different countries direct their behavior, and are moved to act in certain culture-specific ways. ${ }^{42}$ Respondents gave ratings to over fifty words and phrases like "humble”, "moderate”, “clean”, "national security”, "reciprocation of favors", "devout”, "respect for tradition”. These, Schwartz arranged under three headings responsive to "universal requirements": that is, answering to our human needs as individual organisms; second, governing our successful relations with our fellows; and, thirdly, enabling the group or society to function as a whole. Under these three, he arranged Ten values (e.g., "power", or "stimulation") displayed in a pie-chart so as to bring out their relation to each other, adjacent values being nearly similar (and so judged by the scaling technique, "Smallest Space Analysis”). On the pie chart were arranged all fifty-six values which respondents were asked to rate, itself divided into ten sections, and these grouped into four "higher order value systems" (e.g., "self-transcendence”, "self-enhancement”). The four were determined to be "nearly universal," while even in the Ten "many people across contemporary societies recognize value types". "The basic structure... points to the broad

40 Quoted, Hofstede (2002a) 1355, again in (2002); (1994) ix, seeing himself as "spiritual parent" to such studies; a survey of his reception in Berry et al. (2002) 399-401; Smith et al. (2006) 37; and McSweeney (2002) and (2002a).

41 McSweeney (2002a) 1366f. (where in my opinion national character does not permit the prediction of individuals' behavior, only the likelihood of group behavior); further, 1369, destruction of Hofstede's treatment of patterns of labor unrest in several countries.

42 Schwartz (1994) 20ff.; for a slightly fuller definition of "values" see Schwartz (2007) 170f., in a chapter extending his 1994 article into Europe's recent survey; for the history of the term, which most often uses the term or synonyms for "normative" and "belief”, see Parsons and Shils (1962) 390, 395. 
underlying motivations that may constitute a universal principle that organises value systems." 43

As has been true of other assessments of foreign cultures beyond that of the prime investigator, respondents to questions have had to be found in the most convenient quarters; so, for Schwartz' work, about four-fifths were university students and school teachers (the remainder, without further description, being simply "general adults"). In Britain, they were 158 students; in Italy, 200 teachers and 350 students all from Rome; in Indonesia, 263 students (eleven ten-thousandths of one per cent); in India, 200 students (in a population of more than a billion); and so forth. How inadequate the samples were to represent their respective populations is all too clear; and more needs to be said on this point (chap. 3 §). Nevertheless, the publication has been described as "the most influential and respected in the field". ${ }^{44}$

This same assessment of Schwartz' work goes on to add that it is notable principally in its "assumption (which is supported by research) that all people, everywhere, have the same values but differ in terms of the relative importance they place on each value". To say this is to concede an infinite variety in ways of life, such as historians indeed discover; for among every people can be found, no doubt, some minority who hold as a value one of the proposed Ten "motivational values" that everyone else repudiates: for example, "Hedonism... pleasure and sensuous gratification for oneself" as "a goal... that serves the interests of some social entity". ${ }^{45}$ It is no more odd to include this, describing (let us say) the Puritan Colonies of the seventeenth century, than to exclude "God-fearing" as a value, which would figure in the favored list of any number of populations today, not to mention the company on the Mayflower. Its absence in fact inclined Schwartz to invent an Eleventh value, of a rather watery consistency ("spiritual life, inner harmony") which might or might not be added. ${ }^{46} \mathrm{~A}$ Twelfth would be the personal and societal goal of leaving behind a large progeny: therefore "Reproduction" which is found everywhere and across all time. There must be room, too, for the personal and societal goal of renown beyond mere acceptance: therefore, "Approval” or enjoyment of general good repute as a Thirteenth. ${ }^{47}$ I return to "Approval" a little later.

In the end, research into "personology" seems to me to have been fairly described by Seymour Epstein, quoted above. It is capable only of "predicting most of the people much of the time". Limitations on understanding pervade the field. Nothing better than Epstein's dictum appears possible in the absence of any such rules as those that

43 Schwartz (2007) 176.

44 Rohan (2007) 1009f.; criticisms in Smith et al. (2006) 39f., 46, 77.

45 Quoted, Schwartz (1994) 21f.

46 Schwartz (1994) 23.

47 Among his Ten, Schwartz includes "Achievement: Personal success through demonstrating competence according to social standards" - which may indeed help in the attainment of fame, but is different. 
Newton discovered in the universe. Indeed, "structure", the very term in English or other languages in which personality is normally described in realist fashion, betrays in the observer's mind a mental image of a more or less simple, comprehensible design - an inanimate thing; but nothing of the sort has so far been discovered. ${ }^{48}$ If instead personality were thought of not as a Rubik's cube but as a well-populated Petri dish, the mental image would allow life itself to control understanding, and with life, change. This, of course, historians would prefer; this is what they work with. There are consequences in modes of thinking.

The labors of Terman, Hofstede, Schwartz and many others in search of universals, using ever-grander samples and more elaborate quantification, have produced at least one clear finding. They have revealed (and it is very striking) an increasing homogeneity of values and behavior in the developed world of telecommunication, university education, desk-jobs, business attire, and associated features of modern life on which research commonly reports. They are all contemporary since psychology with any pretense of being a science can operate only on the living. There are, however, societies like fossils still alive but properly belonging only to the past, which allow a kind of time travel. A hint of what can be learned from them may be found through trait-analysis of Maasai, spoken by the people of that name in East Africa. This lexical study by Gerard Saucier "indicates a high degree of generalizability in the single-factor structure of a highly traditional culture.” That is, personality is more easily subsumed under a very small number of traits, to the extent it is "tighter (more strict)"; and this can be demonstrated in the range and number of words describing "character/virtue”, "competence/status". ${ }^{49}$ Although at the moment Saucier's choice of subject appears to be unique, ${ }^{50}$ it allows the conjecture that in long-past centuries such a phenomenon was more the rule than the exception. The number of detectible personality "superfactors" is thus not fixed but varies at different points in time and complexity of civilization.

For this possibility there is in fact some support. Psychologists using other methods have shown how qualities seen in a given society simply as socially desirable or the opposite can, by researchers, be bundled together notionally to yield a one-factor model of personality. ${ }^{51}$ This fits with what Harry Triandis and others

48 As a reminder, no doubt needless, that psychologists think about the mind and its operations in a misleading way, as "structure" and "constructed”, see, e.g., Cosmides et al. (1992) 91 ("architecture”, “structure”), Mischel and Shoda (1995) 253, Caspi (1998) 312, 316f., 323, and passim, Löckenhoff and Costa (2007) 115, Schütz and Vater (2007) 994, or Roberts (2009) 139; also above, n. 24.

49 Personal communication (2011) from Saucier, by permission.

50 The Maasai study in Saucier and Goldberg (2006) 272, referring to Saucier's unpublished paper of 2006 and instancing a number of studies or proposals of a Big One structure. McRae and Terracciano (2005) 548 believe "no preliterate cultures have been examined" (ever).

51 Notice the item "Negative Valence”, NV, i.e., simply a general and unexplained disapproval, counted among the Big Seven cited above at n. 26 and in Zhou et al. (2009) 378. 
found in forager or hunter-gatherer groups - populations exhibiting a far narrower range of relationships for members to choose from, therefore a limited range of roles and expectations, therefore far simpler value systems and correspondingly effective group-pressures. ${ }^{52}$ The !Kung of the Kalahari serve to illustrate this at one end of a spectrum, at the other end of which lies our modern city life. In contemporary cultures, simple means poor, complex means rich; rich means able to look around and create one's own life's situation - as is easier to do in the United States, Canada, and Western European countries than in the "collectivist" world of Asia, Africa, and Latin America. ${ }^{53}$ Something of history is implied; the industrial revolution and the development of capitalism are implied; a fourth dimension is rendered in three. ${ }^{54}$

Richard Shweder and Maria Sullivan may serve to conclude my sketch on psychologists' search for rules and universals. The two not only find many and undeniable differences among living societies, they even extend the impact of those differences into the individual personality.

While researchers in cultural psychology are still alert to the possible existence of cross-cultural empirical generalities, which might be derived from comparative research, new presumptions have emerged, e.g. that cultural and institutional factors particular to a population may have a major impact on the processes of psychological functioning and human development, and that local factors of a particular environment typically interact with more widely distributed factors to produce diverse outcomes. ${ }^{55}$

In this opinion may be seen a return to the question put by Auguste Comte in 1852 and asked again by Gordon Allport a century later:

\footnotetext{
How can the individual be both a cause and a consequence of society? That is to say: How can his nature depend indisputably upon the prior existence of cultural designs and upon his role in a predetermined social structure, while at the same time he is clearly a unique person, both selecting and rejecting influences from his cultural surroundings, and in turn creating new cultural forms for the guidance of future generations? ${ }^{56}$
}

And the assumption here is just what Robert Hinde proposed, quoted at the beginning of this chapter: everything that constitutes the individual as such is derived from the surrounding community ("norms, beliefs, and values", as he says, to which other psychologists and anthropologists would add artifacts and everything that makes up a "shared way of life"). The community, so far as it is shared and has boundaries to

52 Triandis (1989) 508ff.

53 Newson et al. (2007) 462; Hofstede (1994) xii; or Triandis (1995) 25 ("affluence leads to individualism”); Kim et al. (1994) 1.

54 Recognized by Kashima and Kashima (2003) 125, while (127) pointing out problems in the idea.

55 Shweder and Sullivan (1993) 501.

56 Allport (1954) 9. 
define it, is in turn the sum-total of the individuals within it. And as they behave, so is their community constituted. "Psyche and culture make each other up."

To explain the origins of personality a little further: let it be said first that historians - except in biography, and then, with reserve - cannot follow psychologists very far. They cannot profit from the discussions in which Comte and Hinde were engaged, or join in, simply from lack of evidence in such depth as personality studies assume. They cannot be concerned with the debate between the so-called "constructionists" who magnify the culture-added parts of personality, and seem to have the better of the argument, versus "geneticists" who focus on inherited tendencies. ${ }^{58}$ Geneticists in fact, beyond basic or hard-wired urges, instincts, or drives governing reproduction, hunger, social relations, and so forth, can point to our latent capacities, the linguistic being the most obvious; and still further, in newborns, and therefore individually innate, personality asserts itself to make a child easily cared for, or alertly responsive, or otherwise unique at birth. ${ }^{59}$ Thereafter successive stages of maturation have been distinguished by (among others) Piaget and Kohlberg. These stages, at least in part and in sequence, are also innate. ${ }^{60}$ The balance, then, between constructionist and geneticist is hard to strike; the questions are too detailed and complicated for clear answers. They can't be applied, anyway, as models in the understanding of an election, let us say, or a literary trend or a strike-vote.

However, I do see possibilities of profit for historians in what psychologists know about traits and values, and the data-base on which their study must depend. I pursue these questions for their bearing on motivation, my prime target: "why do we do what we do?"

Character or behavioral traits, as was recalled above, are ordinarily taken to exclude mental powers such as overall "intelligence” or perfect pitch or photographic memory, but they include a huge range of such things as honesty, aggressiveness, shyness, introversion, curiosity, optimism, sensitivity, generosity, or conscientious-

57 Quoted, R. Shweder in Kitayama et al. (2007) 137; re-phrased by Kim et al. (1994) 5, or Kitayama et al. (2007) 139, “a dynamic process of mutual constitution emerges between culture and self”; or Lehman et al. (2004) 689, "psychological processes influence culture. Culture influences psychological processes". On artifacts also, and tools, technology, and the like as elements of a culture, see, e.g., Triandis (2007) 62; "shared way of life”, Berry et al. (2002) 225.

58 Opposing views about what is acquired vs. genetic open up a million references, e.g., Pervin (1994) 103ff. or Triandis (1989) 507 regarding the species, or Molitor and Hsu (2011) 94 regarding the individual.

59 On urges governing “the major part of man's activities," see, e.g., Malinowski (1947) 102, Caspi (1998) 312, 340, 342; Roberts (2009) 143, "children are born with a wide variety of temperamental starting values”, and to the same effect, Molitor and Hsu (2011) 94; and Pervin (1994) 104 likewise sees "temperament" as innate.

60 On the complexity of our genetic selves and repudiation of the Standard Social Science Model (SSSM) of our make-up, see (persuasively) Cosmides (1992) 28, 93-99. 
ness ${ }^{61}$ Control of traits like these, to restrain or stimulate them, falls under the social instinct. In everyday terms, all of us want the approval of those we live among - if not approval, then acceptance, or if approval, then better yet: applause and good repute. This was proposed on an earlier page as one of the goals to be expected in every population of the present or past, a "superfactor" - and not without good support. Darwin pointed out the "powerful stimulus to the development of the social virtues, namely the praise and the blame of our fellow men," and traced "the love of approbation and the dread of infamy" to its instinctual basis and the logic of survival. ${ }^{62}$ Robert Hogan too looked to our hard-wired dispositions: "The problems of achieving status and maintaining peer popularity are biologically mandated"; and more inclusively, Peter Verbeek declared, "Sociality defines our species. The need to belong through interpersonal attachments is a fundamental motivation." 63

Just how fundamental is evident not only in the remarkable body of observations produced for us by observing chimpanzees, bonobos, and rhesus monkeys and matching from among them all those acts and gestures which serve Homo sapiens in getting along with co-specifics - including ingratiation and deference and status-recognition. No, we can look beyond close relatives, beyond primatology, to see inborn social reflexes at work: we can look even among insects, just where Aristotle chose his illustrations, among ants, wasps, bees.

Add, cockroaches. Psychologists have noticed that people doing a job sometimes did it better if they had an audience, and sometimes did it worse. How come? Robert Zajonc wondered if the answer lay in their consciousness of competence. People quite sure that they could perform well rose to a higher level when they knew others were watching, but those not so sure just made more mistakes. To understand this paradox in simpler terms, Zajonc set 72 female cockroaches to run through an easy maze, and then through a tricky maze, being watched sometimes through a glass partition by other cockroaches, or sometimes accompanied by a co-specific; and the females

61 Defining "traits" such as neuroticism, see, e.g., the view not widely shared that they are "genetically based biological dispositions", so Löckenhoff and Costa (2007) 116 or Bergman and Magnusson (1990) 6; “disposition" equated with "tendency”, A. Tellegen quoted in Roberts (2009) 140; or found in the "ordinary" sense of my own understanding, in Pervin (1994) 108, Asendorpf and Weinert (1990) 193ff., Schütz and Vater (2007) 994f., Costa and McCrae (2009) 299f. On "aggressiveness”, see, e.g., Bergman and Magnusson (1990) 5, Asendorpf and Weinert (1990) 194f., or Brody (1994) 420; "shyness" and "timidity", e.g., Aken and Asendorpf (1996) 205f., Asendorpf (1999) 227, or Bergman et al. (2003) 137; on "intelligence" as a trait, see Mischel (1968) 35, 146 noted in Epstein (1979) 1098 and (1984) 222; also Caspi (1998) 322 and Oishi et al. (2008) 312; or generosity (altruism) in Trivers (1971) 45ff. (I distinguish between culture-bound traits as intended by Hofstede and his like, from genetic social characteristics of our species, of which I say more in chap. 3).

62 Darwin in The Descent of Man, quoted in Verbeek (2006) $424 \mathrm{f}$.

63 Robert Hogan quoted in Smith et al. (2006) 131; Verbeek (2006) 423, 429, with extended observations of child-rearing years and experiences, and the "socioemotional and cognitive framework associated with the deep structure of communal sharing relations". 
slowed down in the easy maze when they were conscious of having company, so to speak, and reacted to it, but handled the tricky maze more quickly with an audience, whether of one or many. The suggested explanation lay in arousal. The females knew they were acting out for others; what others thought of them distracted and excited them, with different consequences depending on their confidence in their performance. ${ }^{64}$

The co-specifics whose approval humans most and first desire are their parents, especially their mother, generally followed by another close member of the family, whether uncle or older sibling or grandparent; and then by more distant kin, neighbors, and generally acknowledged authority figures who embody the entire society as a school of trait-learning. "Early development, punishment and reward are delivered not only by the entities themselves," whether a kin-group or a nation, "but by parents and other elders and peers, who usually embody the social conventions and ethics of the culture to which the organism belongs" - so, Antonio Damasio, whose work will need mention later. ${ }^{65}$ The social instinct is ever at work, in confined or public settings. From the instant of birth, we oblige, we observe and imitate, always to win approval. In the process our individual character is formed out of our innate potential; in the process and in the aggregate, the character of an entire people is formed.

So much for the role and importance of the social instinct, both in giving cohesion to a society and its ways, and in shaping the individual's behavioral tendencies. But further: measurement of these tendencies (as historians would like to know) can have predictive application. For example, mothers' forming of will-power in their little children, as Mischel noted, increased the children's likelihood of success in school. A number of other studies have been mentioned above, showing long-term costs or benefits nicely correlated with lifetime outcomes in marriage, social relations, career or health. ${ }^{66}$ In brief, and quite obviously, how you are shaped as a child determines to a very significant degree how you will shape your course of life and the society around you - since these two are one.

These findings are clearly relevant to the historical discipline, but they can be specially illustrated from the work of ethnologists, who are interested in the whole picture. Jean Briggs and Esther Goody have closely observed (in Canada and Ghana) how mothers behave with small children. ${ }^{67}$ Their object as always anywhere was to prepare the infant for success in life, which at the beginning means winning the mother's approval, and then in time most likely includes a male parent's as well,

\footnotetext{
64 Blascovich (1999) 68f., 75f.; Mendes (2007) 898; and studies among Homo sapiens to show better performance if watched, Leary and Allen (2011) 44.

65 Damasio (1994) 174.

66 Mischel and Gilligan (1964) 412f.; Ozer and Benet-Martínez (2006) 409, 412, a personality trait is a "strong predictor" of adult career success; and above at nn. 3f. and 22f.

67 Briggs (1970) and Goody in several publications, best in (1991) 106f., illustrating how dependence on group acceptance and good opinion is taught in childhood in a Ghanaian population, and in Japan.
} 
and older siblings and grandparents and so outward from the core family to a wider and wider community, as maturation (which is socialization) continues - always, for approval, though least evidently in individualistic cultures like the American.

In a collectivistic one like the Japanese, the child is "carefully taught", Goody explains,

to want to participate energetically in groups based in the neighborhood, school and work, and to seek the good opinion of group members. In order to be sure of acceptance s/he learns to be highly sensitive and responsive to the feelings and wants of the other members of these groups. However, as long as we work within definitions of particular cultures it is not possible to make very interesting observations about learning, since what is learned differs so widely. We tend to find ourselves considering the learning of norms per se. Is it possible to go beyond this relativity?

- and Goody engages herself in the attempt to discern larger truths, on the model of physics. ${ }^{68}$

Historians, however, are easily satisfied with less. What the ethnologists notice (especially Goody drawing a number of good comparisons) is quite interesting enough; the conclusions are as thought-provoking as they are acutely observed, for example: "many, probably all, societies consider that one of the major responsibilities of parents is the moral education of their children... based on precept, parable and proverb which specify how a 'good Gonja/Ashanti... man/woman' ought to behave... There are constraints of affect built up in the nurturance relationship, as well as constraints arising from the control the parent has over access to critical resources." 69

Socialization of course continues beyond childhood. Something more will have to be said on proverbs in chapter 3, and, in chapter 2, on continuing moral lessons in adulthood that Briggs and Goody don't discuss. Their focus on childhood, however, is well chosen to bring out the emotional nature of socialization; "the primacy of emotion in morality," from parental hugs and tidbits to more adult demonstrations of approval for good behavior and the accompaniment of words to describe that behavior, so that the moral vocabulary is filled with feelings. ${ }^{70}$ It is feelings, turning us toward or away from something, that are internalized and can motivate well-taught conduct. There is no reason to think motivation was governed or should be explained in any different way in times past, to which historians turn. ${ }^{71}$

68 Goody (1991) 106f.; centrality of teaching, 109ff., 114ff., 121ff.

69 Goody (1992) $13 f$.

70 Quoted, Verbeek (2006) 426, citing publications, and again, 439. See also Murray (1951) 454, "the seeds of attitudes (which are basically affective, rather than cognitive) are implanted in childhood"; and Hinde (1996) 371 instances many studies to show that Japanese maternal style invites closer affect and attention and uses more affect-salient speech to the child than American or European style, i.e., in a collectivistic society which in my view is the more likely character of societies everywhere, in the past (above at nn. 37f.).

71 Aversive/appetitive emotions accompanying immoral or "morally acceptable behaviors", in Bar- 
To learn so much from psychology about traits and their relation to the core character of the surrounding society is very welcome, but a reminder, too, that there is no perfect fit between what historians need to do, and psychologists. The disjunction may be recalled, from an earlier page, between traits and values. We know that the former only describe conduct but "that values influence behavior"; "values guide action". ${ }^{72}$ To get beyond observation into the mind of the person or people observed, and so to understand motivation, historians lack the living samples that psychologists can question. They must depend on written sources of which the most obviously promising are letters, diaries, memoirs, and in short, biography;but even a work of this genre, if it is to satisfy, must give some attention to the interaction between protagonist and supporting cast. Without the latter, the protagonist has no more significance than Hamlet at one of his soliloquies. For more serious historical interests, increasingly for a century and more it has been the life and actions of whole classes or populations that count; and, for their interpretation, the tricks of biography are of no use; of no use, much of psychology as a discipline, for all its achievements, in which it is the individual that is explained, not groups or masses. What is instead most helpful to historians is what psychology has to say about values. Its findings offer the best chance of reading the mass mind that lies behind mass behavior.

nett (2007) 586f.; Harris (1995) 474 or Hinde (1996) 371f. on Asian child-training suited to a collectivist culture.

72 The first quotation is from Bond (1994) 74, where the author is concerned also to show how "to integrate the cultural dimension into theories of behavior," and, as values like behavior differ according to culture, values are "the mediating variable of interest that connects to culture"; the second quotation is Schwartz (2007) 171, with good explanation of values as goals, as means of measuring proposed action, and as motivational, $170 f$. 